Pesq. Vet. Bras. 35(9):795-800, setembro 2015 DOI: 10.1590/S0100-736X2015000900005

\title{
Influência do estrógeno e do interferon $\gamma$ sobre a expressão da indoleamina-2,3-dioxigenase em cultura de células de placenta e embriões de ratas Wistar ${ }^{1}$
}

\author{
Maria Letícia Baptista Salvadori² ${ }^{2}$ Pedro Kastein Faria da Cunha Bianchi², \\ Bernadete de Lourdes Liphaus ${ }^{3}$, Érika Zolcsák Sousa ${ }^{2}$, Renata Santos Silva ${ }^{2}$ \\ e José Roberto Kfoury Junior ${ }^{2 *}$
}

\begin{abstract}
Salvadori M.L.B., Bianchi P.K.F.C, Liphaus B.L., Souza E.Z., Silva R.S. \& Kfoury Júnior J.R. 2015. [Estrogen and interferon $\gamma$ influence on 2, 3 indoleamine-dioxygenase expression in culture of placenta and fetal cells from Wistar rats.] Influência do estrógeno e do interferon $\gamma$ sobre a expressão da indoleamina-2,3-dioxigenase em cultura de células de placenta e embriões de ratas Wistar. Pesquisa Veterinária Brasileira 35(9):795800. Setor de Anatomia, Departamento de Cirurgia, Faculdade de Medicina Veterinária e Zootecnia, Universidade de São Paulo, Av. Prof. Dr. Orlando Marques Paiva 87, Butantã, São Paulo, SP 05508-270, Brazil. E-mail: jrobertok@usp.br

The indoleamine 2,3-dioxygenase (IDO) is an enzyme responsible for catabolizing the tryptophan. Its presence in the placental uterine environment is related to immunological tolerance to the semi-allograft because it prevents proliferation of maternal immune cells, either by the lack of this amino acid or by the action of its catabolites, such as the quinolinic acid, which is particularly toxic for T lymphocytes. Little is known regarding the influence of hormones and cytokines on the expression of IDO in the maternal fetal interface. Therefore, the hypothesis that some hormones and interleukins present in uteroplacental region could have an effect on the expression of IDO on cultured cells was tested. Cells derived from the fetal maternal interface from Wistar rats were kept in culture and supplemented with estradiol and interferon- $\gamma$. Expression of the enzyme was assessed by flow cytometry at periods of 4, 24 and 48 hours and confirmation of the presence of protein by immunohistochemistry. The results showed an increasing of IDO expression after the addition of estrogen $(9.03 \pm 0.81$ to $11.25 \pm 0.25)$ and interferon $-\gamma(9.03 \pm 0.81$ to $20.43 \pm 0.60)$. The effect of interferon- $\gamma$ was expected as reported in the literature, however, elevated IDO expression by estrogen represents new information on possible mechanisms involved in the enzyme activation. These findings could provide a better understanding of IDO contribution on maternal-fetal tolerance and may collaborate to future therapeutic modulation of this enzyme.
\end{abstract}

INDEX TERMS: Indoleamine 2,3-dioxygenase, maternal-fetal tolerance, placenta, embryo, flow cytometry.

RESUMO.- A indoleamina 2,3-dioxigenase (IDO) é uma enzima responsável por catabolizar o aminoácido triptofano. Sua presença no ambiente uterino placentário está

\footnotetext{
${ }^{1}$ Recebido em 22 de maio de 2015.

Aceito para publicação em 13 de setembro de 2015.

${ }^{2}$ Departamento de Cirurgia/Setor Anatomia, Faculdade de Medicina Veterinária e Zootecnia (FMVZ), Universidade de São Paulo (USP), Av. Prof. Dr. Orlando Marques de Paiva 87, São Paulo, SP 05508-270, Brasil. *Autor para correspondência: jrobertok@usp.br

${ }^{3}$ Laboratório de Investigações Médicas 36 (LIM 36), Instituto da Criança do Hospital das Clínicas, Faculdade de Medicina, Universidade de São Paulo, Av. Dr. Enéas Carvalho de Aguiar 647, São Paulo, SP 05403-000, Brasil.
}

relacionada à tolerância imunológica ao semi-aloenxerto, pois impede a proliferação de células imunológicas maternas, seja pela falta do aminoácido, ou pela ação de alguns catabólitos oriundos da quebra do triptofano, como o ácido quinolínico, que é tóxico principalmente para os linfócitos T. Pouco se conhece sob a influência de substâncias (hormônios e citocinas) presentes na interface materno fetal e a expressão dessa enzima. Por esta razão, formulou-se a hipótese de que hormônios e interleucinas presentes na região uteroplacentária poderiam exercer algum efeito na expressão da IDO. Células oriundas da interface materno fetal de ratas Wistar foram mantidas em cultivo, onde 
receberam suplementação com estradiol e interferon- $\gamma$. A expressão da enzima foi avaliada pela técnica de citometria de fluxo nos períodos de 4, 24 e 48 horas e confirmação da presença proteica por imuno-histoquímica. Os resultados mostraram um aumento na expressão de IDO após a adição de estrógeno $(9,03 \pm 0,81 / 11,25 \pm 0,25)$ e interferon- $\gamma$ $(9,03 \pm 0,81 / 20,43 \pm 0,60)$. 0 efeito do interferon- $\gamma$ já era esperado como relatado na literatura, contudo, a elevação da expressão da IDO pela adição do estrógeno constitui nova informação sobre possíveis mecanismos envolvidos na ativação da enzima. 0 melhor esclarecimento desses achados poderia contribuir para uma melhor compreensão da participação dessa enzima na tolerância materno-fetal e para uma futura modulação terapêutica da mesma.

TERMOS DE INDEXAÇÃO: Indoleamina 2,3-dioxigenase, tolerância materno-fetal, placenta, embrião, citometria de fluxo.

\section{INTRODUÇÃO}

O comportamento biológico no período gestacional agrega várias mudanças imunoendócrinas visando à tolerância aos antígenos paternos presentes no feto ( Kolb et al. 1984, Croy et al. 1987, Mailankot \& Nagaraj 2010). Um dos mecanismos de tolerância materna ao semi-aloenxerto é mediado pela enzima indoleamina 2,3-dioxigenase (IDO) (Pereira et al. 2005, Sedlmayr et al. 2014).

A IDO é expressa por um vasto grupo de células, incluindo as células trofoblásticas (Munn et al. 1998). Age reduzindo a concentração do aminoácido triptofano pelo seu catabolismo, como ocorre no micro ambiente placentário (Mellor \& Munn 2001, Li et al. 2015). Células presentes neste microambiente com alta expressão de ID0, podem ser induzidas a um estado apoptótico pela carência de triptofano e pela ação de catabólitos tóxicos como a quinurenina, levando, principalmente as células $\mathrm{T}$ ativadas a apoptose, favorecendo a formação de um ambiente tolerogênico (Munn et al. 1998, Mellor et al. 2003, Saito et al. 2007).

Sabe-se que a atividade bioquímica e funcional da IDO é estimulada, principalmente, pelo interferon- $\gamma(\mathrm{INF}-\gamma)$, pois o mesmo induz a transcrição da proteína (Strehlow et al. 1993, Kudo et al. 2004, Muller et al. 2005, Sugimoto et al. 2006).

Contudo, pouco se conhece sobre a relação entre o estrógeno, um dos principais hormônios presentes durante o processo gestacional, e a IDO. Por isso, sabendo que o estrógeno altera o padrão de citocinas produzidas pelas células imunológicas e, que a IDO é estimulada por algumas destas citocinas, como o IFN- $\gamma$, buscou-se, com este trabalho, verificar se a expressão da enzima é influenciada por tais substâncias, avaliando o comportamento da expressão da IDO em culturas de células presente na interface materno fetal de ratas Wistar prenhes.

\section{MATERIAL E MÉTODOS}

As amostras utilizadas foram oriundas de gestações de fetos normais coletados de animais de biotério (ratas Wistar com 85 dias de idade). Devido às diminutas dimensões dos embriões e placentas, as células foram extraídas e analisadas em conjunto (células do embrião + células placentárias + células imunológicas), uma vez que não foi possível separá-los com precisão. As amostras foram separadas em três "pools" de cinco animais, a fim de se con- seguir um número mínimo suficiente de células para a realização das culturas e análises propostas. 0 presente projeto encontra-se de acordo com os princípios éticos de experimentação animal da Comissão de Ética para uso de animais da Faculdade de Medicina Veterinária e Zootecnia da Universidade de São Paulo (nำ1742/2009).

Cultivo celular. Os tecidos uterinos, as placentas e os embriões foram seccionados e dissociados mecanicamente. Para isso, utilizou-se uma malha de aço de 150 mesh de diâmetro acoplada a uma placa de petri de $50 \mathrm{ml}$. Após esse procedimento, o conteúdo presente no fundo da placa foi suspenso em meio de cultura (D-MEM-Sigma Aldrich, USA), com 10\% de SFB (Invitrogen, USA) e, submetido à centrifugação $\left(700 \times\right.$ g, por 5 minutos a $\left.20^{\circ} \mathrm{C}\right) .0$ sobrenadante foi descartado e as células foram novamente suspensas em meio de cultura D-MEM (Sigma Aldrich, USA) 10\% SFB (Invitrogen, USA).

Procedeu-se a contagem e a estimativa de viabilidade das células pelo método de exclusão por azul de trypan. Após, as células foram postas em placas de 48 poços numa concentração de $1 \times 10^{6}$ células $/ 250 \mu \mathrm{l}$, sendo incubadas à $37^{\circ} \mathrm{C}$ e $5 \%$ de $\mathrm{CO}_{2}$ até o início das análises.

Os fatores foram adicionados ao início do cultivo e, os tratamentos foram assim constituídos: 1- sem tratamento, somente meio de cultura (D-MEM-Sigma Aldrich, USA); 2-D-MEM + estradiol (E.C.P. $®$ Cipionato de Estradiol Pfizer, Brasil - 0,002 mg/250 $\mu \mathrm{l}$ ) (Xiao et al. 2004); 3-D-MEM + interferon $\gamma$ (Millipore, USA $0,01 \mathrm{mg} / 250 \mu \mathrm{l}$ ) (Gromann et al. 2003, Barrera et al. 2007).

Citometria de fluxo. Nos períodos estabelecidos para as análises, 4,24 e 48hs, as células foram desprendidas das placas e centrifugadas a $700 \times \mathrm{g}$ por 5 minutos a $20^{\circ} \mathrm{C}$. Após, as células $(250 \mu \mathrm{l}$ contendo $1 \times 10^{6}$ células) foram colocados em tubos de citometria (BD, Brasil) e permeabilizadas com $0,3 \%$ de Triton X. Posteriormente foram incubadas com $1 \mu \mathrm{l}$ anticorpo primário anti-IDO (mouse monoclonal IgG-Millipore, USA), por $30 \mathrm{~min}$. Seguindo o protocolo, as células foram lavadas e ressuspensas em PBS 0,1M, sendo incubadas com $1 \mu \mathrm{l}$ anticorpo secundário goat anti-mouse conjugado com FITC (goat anti mouse IgG, Dako, Dinamarca) por 30 min protegido da luz. Por fim, as células foram novamente lavadas, resuspendidas em $300 \mu$ l de tampão FACS (BD, Brasil) e analisadas em citômetro de fluxo (FACS Calibur - BD-Cytek, USA). A viabilidade celular foi novamente verificada utilizando o Kit Live/Dead cells (Fixable Violet Dead Cell Stain Kit-Invitrogen, USA). Controles para autofluorescência e marcação cruzada pelo anticorpo secundário foram realizados com grupos contendo somente células e grupos contendo somente células e o anticorpo secundário, respectivamente.

Imuno-histoquímica. Cortes de $2 \mu \mathrm{m}$ foram desparafinizados em xilol ( $2 \times 10 \mathrm{~min})$, reidratados em uma série decrescente de etanol (etanol 100\%, 2×5 min; etanol 95\%, 5 min; etanol 70\%, 5 min) e lavados em água destilada (5 min). A recuperação antigênica ocorreu incubando-se em solução tampão citrato 10nM, pH 6, por 5 minutos, à temperatura ambiente, seguida por 15 minutos de irradiação em micro-ondas em potência alta (560W). Em seguida, as lâminas foram resfriadas por 20 minutos à temperatura ambiente e lavadas em água destilada por 5 minutos. A atividade da peroxidase endógena foi bloqueada incubando-se as lâminas em peróxido de oxigênio a $1 \%$ diluído em metanol por 30 minutos, em freezer. As mesmas foram lavadas em tampão ICC/Triton X 0,3\%, pH 7,2-7,4 (PBS $+3 \mathrm{ml}$ de Triton $\mathrm{X} /$ litro), três vezes de 5 minutos, e incubadas em caseína (Protein block Serum-Free, X0909, Dako North America, Inc.) por 20 minutos, à temperatura ambiente, para bloquear sítios de ligações inespecíficas. Após a secagem do bloqueador, o anticorpo primário IDO-Clone 10.1 (mouse monoclonal IgG-Millipore, USA) foi utilizado na diluição de 1:600 e incubados overnight a $4^{\circ} \mathrm{C}$. Os controles negativos foram incubados com um anticorpo monoclonal irrelevante, isotipo específico, em concentração igual 
ao do respectivo anticorpo primário (Coulter Immunotech Diagnostics, Alemanha). Após lavagem em tampão ICC por três vezes de 5 minutos, o anticorpo secundário biotinilado (Biotinylated Link; kit Dako LSAB + System-HRP, K0679, Dako North America, Inc) foi adicionado e as lâminas incubadas por 15 minutos à temperatura ambiente. As mesmas foram lavadas em tampão ICC por três vezes de 5 minutos e incubadas com o complexo estreptavidina-peroxidase (Streptavidin-HRP; kit Dako LSAB + System-HRP, K0679, Dako North America, Inc.) por 15 minutos, à temperatura ambiente. Após lavagem com tampão ICC por três vezes de 5 minutos, a reação de imuno-histoquímica foi iniciada com o cromógeno DAB (DAB + Subtrate buffer; kit Dako LSAB + System-HRP, K0679, Dako North America, Inc) por aproximadamente 60 segundos. Em seguida, as lâminas foram lavadas duas vezes de 5 minutos em água destilada, rapidamente contracoradas com hematoxilina (aproximadamente 30 segundos), lavadas em água corrente por 10 minutos, reidratadas em uma série graduada de álcool $(70 \%, 95 \%$, II e I; 2 minutos cada) e xilol (II e I; 2 minutos cada) e, finalmente, montadas com Permount $^{\circledR}$ (Thermo Fisher Scientific Inc, EUA).

Análise estatística. Utilizou-se o teste ANOVA (one-way) para verificar a significância da variação existente entre as mé- dias de cada fator nos três diferentes pools, seguido pelo teste de TUKEY-KRAMER indicado para localizar qual subgrupo de médias sofreu maior alteração pelo fator adicionado quando comparado ao grupo controle. Ambos realizados com a utilização do software INSTAT3 (Graph Phad - San Diego- USA), sendo os resultados considerados significativos quando $p<0,05$.

\section{Citometria de fluxo}

\section{RESULTADOS}

A expressão de IDO apresentou um aumento significativo 4 h após à adição de estrógeno $(9,03 \pm 0,81 / 11,25 \pm 0,25)$ e $24 \mathrm{~h}$ após a adição de interferon $\gamma(9,03 \pm 0,81 / 20,43 \pm 0,60)$, conforme apresentados no gráfico (Fig.1). Exemplos das aquisições em histograma são mostrados na Figura 2 e, a presença da IDO à interface materno fetal esta demonstrada na Figura 3.

\section{Imuno-histoquímica}

A proteína IDO foi detectada no meio intracelular da região interface materno fetal, por meio da imuno-histo-
INTERFERONP

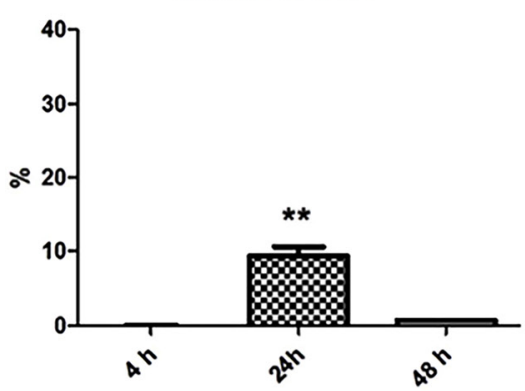

ESTRÓGENO P

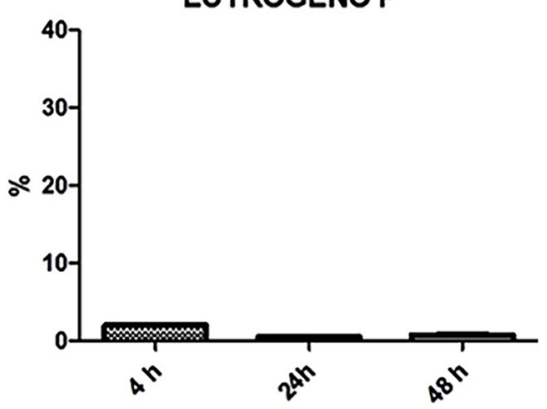

SC P

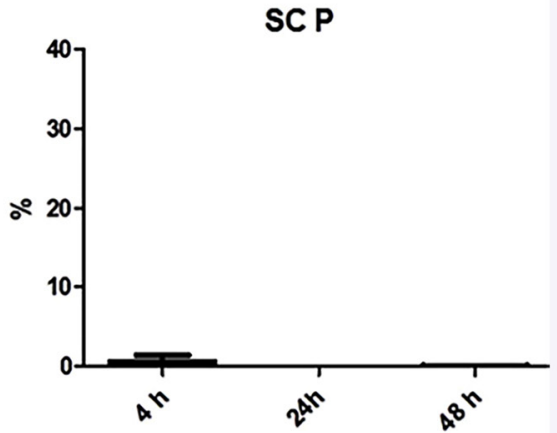

Fig.1. Valores médios e respectivos desvios-padrões da expressão da IDO (\%) em células placentárias e embrionárias de ratas prenhes (seis "pools" de 5 animais) em cultura no período de 4, 24 e 48 horas após a adição de estrógeno e interferon-y.
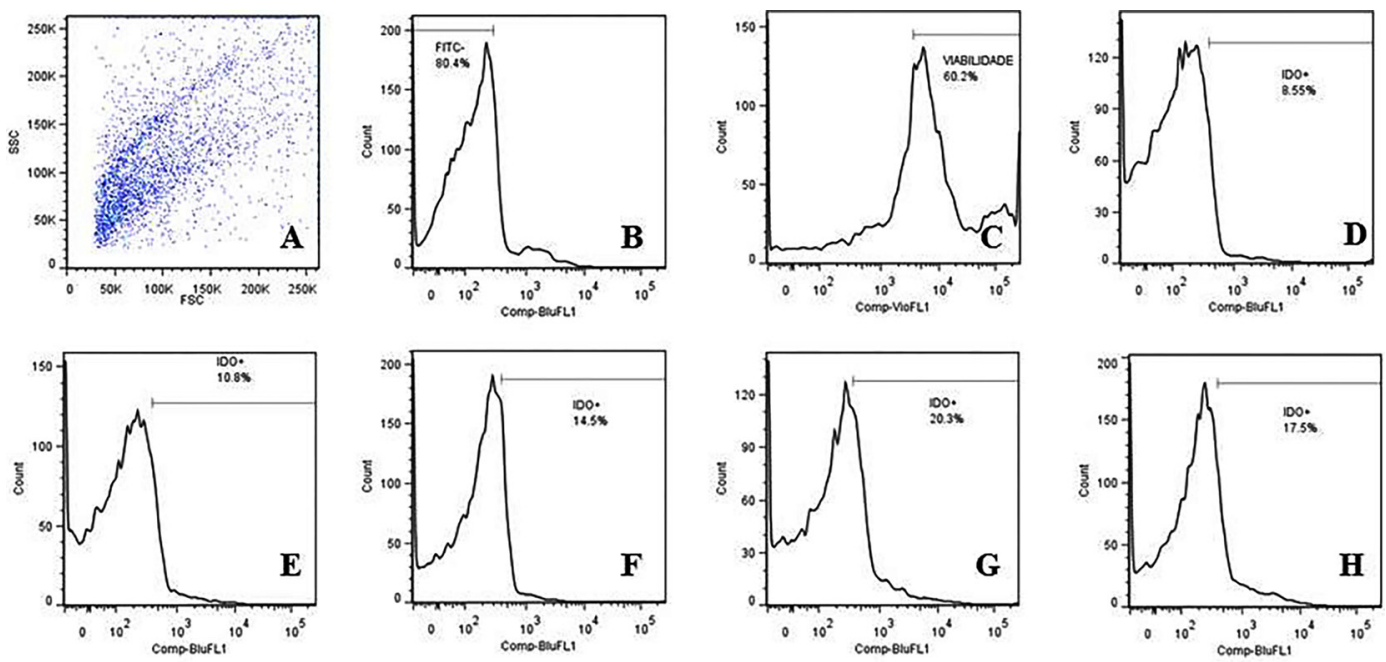

Fig.2. Exemplos de aquisições em histograma da expressão da IDO (\%) em células dos pools de ratas prenhes em cultura no período de $4 \mathrm{~h}$ após a adição de hormônios e citocinas. (A) Perfil morfológico das células de útero, embrião e placenta, (B) FITC, (C) Viabilidade celular, (D) Controle, (E) Estrógeno, (F) Interferon $\gamma$.

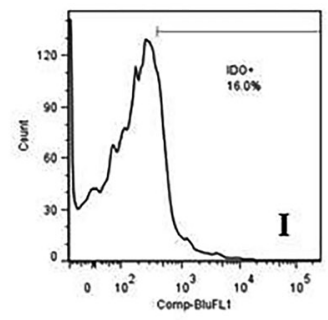



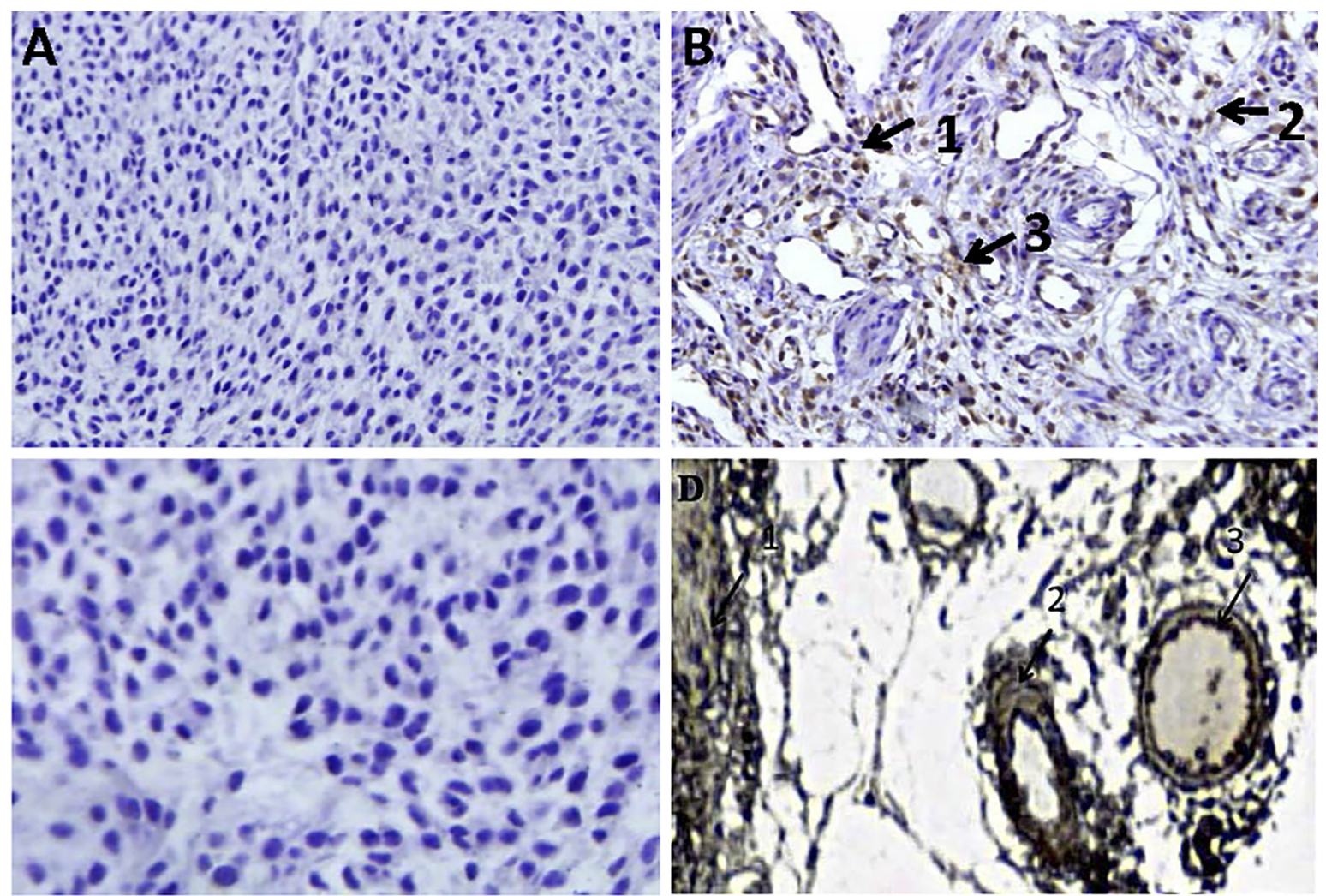

Fig.3. Imuno-histoquímica da região interface materno fetal realizada em dois períodos gestacionais, (A,B) com 13 dias e (C,D) com 6,5 dias de gestação. As imagens A e C são controles negativos e as B e D apontam as regiões com a presença da enzima (IDO).

química realizada em dois períodos gestacionais, 6,5 e 13 dias (Figura 3). Conforme visto nas imagens da imuno-histoquímica a IDO é altamente expressa nas células da região útero placentária em ambos os períodos, porém com maior intensidade no sexto dia gestacional, conforme visto na imagem D. A expressão da enzima é bastante evidente nas células da junção útero placentária, conforme visualizado na figura (D1) e, também, nas células ao redor dos vasos sanguíneos (D2), bem como nas vilosidades presentes na região da interface materno fetal (B).

\section{DISCUSSÃO}

A expressão de IDO apresentou um aumento após a adição de estrógeno, porém, houve um aumento significativo após a adição do interferon $\gamma$.

Durante a implantação embrionária a inflamação é um processo notório, onde existe uma vasta gama de citocinas decorrentes do desenvolvimento desse processo (citocinas pró-inflamatórias), como o interferon $\gamma$. Já é sabido que o IFN- $\gamma$ estimula uma forte atividade bioquímica e funcional da IDO, pois induz a transcrição da proteína (IDO) pela via de sinalização JAK 1 e JAK 2 (Mailankot \& Nagaraj 2010).

A expressão de IDO pelo IFN- $\gamma$ sugere uma modulação da resposta imunológica sinalizada por uma citocina pro-inflamatória, pois a enzima é capaz de atenuar a resposta imunológica em determinado microambiente em que é expressa (Munn et al. 1998), como no caso da interface materno fetal, local com alta reposta inflamatória durante o processo de implantação embrionária, em que a enzima pode atenuar a resposta imunológica contra o aloenxerto, devido a sua capacidade de catabolizar o triptofano, impedindo o acesso das células imunológicas à esse composto, que é vital para a sua sobrevivência e multiplicação. Com isso a interface materno fetal torna-se um local privilegiado, pois pode-se pressupor que a IDO age como um imunomodulador, fazendo parte de um mecanismo de controle da resposta imunológica em determinado microambiente.

Ainda não são conhecidos quais os tipos celulares que expressam IDO na interface materno fetal de ratas Wistar, no entanto, em humanos e camundongos a sua expressão é vista em células imunológicas, como linfócitos $\mathrm{T}$ e células dendríticas (Munn et al. 1999, Mellor et al. 2003, Munn et al. 2004, Hainz et al. 2005 ). Vale ressaltar que a expressão de IDO é maior no inicio da gestação, conforme visto em nosso trabalho pela imuno-histoquímica, em que a expressão de IDO foi maior nos animais com 6,5 dias de gestação (Suzuki et al. 2001).

Ainda, conforme mostrado na imuno-histoquímica, células ao redor dos vasos sanguíneos presentes na região útero placentária expressam consideravelmente a enzima, um fato muito interessante, pois células imunológicas podem estar migrando do endotélio vascular para os tecidos placentários, na tentativa de atacar o concepto. A presença da IDO nesta região pode sugerir que células com alto potencial migratório, como os macrófagos e células dendríticas, possam potencialmente expressar a enzima, colaborando com a tolerância materno fetal, já que tais células são as principais iniciadoras de uma resposta imunológica. 
Supõe-se que no início da gestação a reposta imunológica contra o aloenxerto seja maior, pois o mesmo esta sendo reconhecido pelo sistema imunológico materno como um corpo estranho, por isso, a modulação do sistema imunológico deve ser maior, explicando a alta expressão de IDO nesse período. No entanto o ataque ao aloenxerto não é cessado, apesar de menos severo, durante todo o processo gestacional, sendo necessário que a enzima esteja presente, durante toda a gestação, para proteger o concepto.

0 estradiol exerce seus efeitos nas células que possuem receptores específicos para o mesmo, como os linfócitos B, T, células dendríticas (Tanriverdi et al. 2003). A adição de estradiol em nossos experimentos conduziu a um leve aumento da expressão de IDO. Tal resultado poderia ser justificado devido ao fato de que células da placenta e de embriões com 6,5 dias de gestação apresentam baixo número de receptores disponíveis (Morishige \& Leathem 1972) e, tais receptores já poderiam estar ocupados pelo estradiol endógeno, excluindo a alta influência do estrógeno na expressão de IDO.

No entanto, quando o estrógeno se liga aos seus receptores específicos presentes nas células imunológicas, ocorre uma mudança de perfil, em que as células podem produzir citocinas pró-inflamatórias, dependendo da necessidade e do momento (Krämer et al. 2012), um fato contraditório, pois uma resposta inflamatória poderia causar a rejeição fetal. Porém, citocinas pró-inflamatórias são capazes de estimular fortemente a expressão de IDO, que irá impedir o desenvolvimento de uma resposta inflamatória prejudicial ao processo gestacional.

Conforme visto neste trabalho, o estrógeno tem poder de alterar a expressão de IDO, o que o torna ainda mais importante no microambiente da interface materno fetal, pois pode estar indiretamente relacionado a mecanismos de controle do sistema imunológico materno, agindo pela mudança de perfil das células imunológicas com consequente estimulação da IDO, mecanismo capaz de atenuar as respostas imunológicas maternas contra o aloenxerto.

$\mathrm{O}$ aumento de IDO após a suplementação com IFN- $\gamma$ e estrógeno sugere que as próprias células uterinas ou, que outras células presentes no útero, como por exemplo, macrófagos, células dendríticas, linfócitos entre outras, sofram influência destes fatores. Por isso, estudos em andamento por nosso grupo estão investigando as possíveis células envolvidas (Szekeres-Bartho \& Wegamnn 1996, Druckmann \& Druckmann 2005, Barrera et al. 2007), buscando compreender os mecanismos de ativação e inibição da enzima, na tentativa de modular a expressão da IDO, que poderá ser utilizada, no futuro, em tratamentos de distúrbios gestacionais relacionados à tolerância materno-fetal, além de aspectos como alergia, imunologia de tumores, autoimunidade e infecção por HIV.

\section{CONCLUSÕES}

Considerando os resultados obtidos, foi possível evidenciar um aumento significativo na expressão de IDO quando as células foram submetidas à suplementação com interferon- $\gamma$ e, um discreto aumento com adição de estrógeno, o que permite novas hipóteses para melhor compreensão da participação dessa enzima na tolerância materno-fetal, particularmente em relação a sua interação com hormônios e citocinas presentes no útero gestante.

Considerando os resultados obtidos, verificou-se que o estrógeno e o interferon- $\gamma$ causam alterações na expressão da enzima indoleamina 2,3 dioxigenase.

Tais achados contribuem para o desenvolvimento de novas hipóteses para melhor compreensão das vias em que citocinas (interferon- $\gamma$ ) e hormônios esteroides (estrógeno) atuam para interferir na expressão da IDO nas células da interface materno fetal.

Agradecimentos.- Agradecemos ao apoio financeiro da Fundação de Amparo à Pesquisa do Estado de São Paulo (FAPESP, processo 09/54597-2).

\section{REFERÊNCIAS}

Barrera D., Ávila E. \& Díaz L. 2007. Papel inmunológico de la progesterona en el mantenimiento del embarazo. Revta Invest. Clín. 59:139-145.

Croy B.A., Wood W. \& King G.J. 1987. Evaluation of intrauterine immune suppression during pregnancy in a species with epitheliochorial placentation. J. Immunol. 139:1088-1095.

Druckmann R. \& Druckmann M.A. 2005. Progesterone and the immunology of pregnancy. J. Steroid Biochem. Mol. Biol. 97:389-396.

Grohmann U., Fallarino F. \& Puccetti P. 2003. Tolerance, DCs and tryptophan: much ado about IDO. Trends Immunol. 24:242-248.

Hainz U., Obexer P., Wilkler C., Sedlmayr P., Takikawa O., Greinix H., Lawitschka A., Pötschger U., Fuchs D., Ladisch S. \& Heitger A. 2005. Monocyte-mediated T-cell suppression and augmented monocyte tryptophan catabolism after human hematopoietic stem-cell transplantation. Blood 105:4127-4134.

Kolb J.P., Chaouat G. \& Chassoux D. 1984. Immunoactive products of placenta. III. Suppression of natural killing activity. J. Immunol. 132:2305-2310.

Krämer S.D., Mu L., Müller A., Keller C., Kuznetsova O.F., Schweinsberg C., Franck D., Müller C., Ross T.L., Schibli R. \& Ametamey S.M. 2012. 5-(2-18Ffluoroethoxy)-L-tryptophan as a substrate of system L transport for tumor imaging by PET. J. Nucl. Med. 53:434-442.

Kudo Y., Boyd C.A., Spyropoulou I., Redman C.W., Takikawa O., Katsuki T., Hara T., Ohama K. \& Sargent I.L. 2004. Indoleamine 2,3-dioxygenase: distribution and function in the developing human placenta. J. Reprod. Immunol. 61:87-98.

Li D.D., Yin Y.H., Wu J.Y., Yang Z.Q., Cao H., Zhang Q.L., Guo B. \& Yue Z.P. 2015. Effects of ido1 on mouse decidualization. Mol. Biol. 49:649-657.

Mailankot M. \& Nagaraj R.H. 2010. Induction of indoleamine 2,3-dioxygenase by interferon-gamma in human lens epithelial cells: apoptosis through the formation of 3-hydroxykynurenine. Int. J. Biochem. Cell Biol. 42:1446-1454.

Mellor A.L. \& Munn D.H. 2001. Tryptophan catabolism prevents maternal $\mathrm{T}$ cells from activating lethal anti-fetal immune responses. J. Reprod. Immunol. 52:5-13.

Mellor A.L., Baban B., Chandler P., Marshall B., Jhaver K., Hansen A., Koni P.A., Iwashima M. \& Munn D.H. 2003. Cutting edge: induced indoleamine 2,3 dioxygenase expression in dendritic cell subsets suppresses $\mathrm{T}$ cell clonal expansion. J. Immunol. 171:1652-1655.

Morishige W.K. \& Leathem J.H. 1972. Pregnancy maintenance with corticosterone in protein-depleted rats: a study on fetal protein composition. Endocrinology 90:318-322.

Muller A.J., DuHadaway J.B., Donover P.S., Sutanto-Ward E. \& Prendergast G.C. 2005. Inhibition of indoleamine 2,3-dioxygenase, an immunoregulatory target of the cancer suppression gene Bin1, potentiates cancer chemotherapy. Nat. Med. 11:312-319.

Munn D.H., Sharma M.D. \& Mellor A.L. 2004. Ligation of B7-1/B7-2 by human CD4+ T cells triggers indoleamine 2,3-dioxygenase activity in dendritic cells. J. Immunol. 172:4100-4110.

Munn D.H., Shafizadeh E., Attwood J.T., Bondarev I., Pashine A. \& Mellor 
A.L. 1999. Inhibition of T cell proliferation by macrophage tryptophan catabolism. J. Exp. Med. 189:1363-1372.

Munn D.H., Zhou M., Attwood J.T., Bondarev I., Conway S.J., Marshall B., Brown C. \& Mellor A.L. 1998. Prevention of allogeneic fetal rejection by tryptophan catabolism. Science 281:1191-1193.

Pereira A.C., Jesús N.R., Lage L.V. \& Levy R.A. 2005. Imunidade na gestação normal e na paciente com lúpus eritematoso sistêmico (LES). Revta Bras. Reumatol. 45:134-140.

Saito S., Shima T., Nakashima A., Shiozaki A., Ito M. \& Sasaki Y. 2007. What is the role of regulatory $\mathrm{T}$ cells in the success of implantation and early pregnancy? J. Assist. Reprod. Genet. 24:379-386.

Sedlmayr P., Blaschitz A. \& Stocker R. 2014. The role of placental tryptophan catabolism. Front. Immunol. 19: 200-235.

Strehlow I., Seegert D., Frick C., Bange F.C., Schindler C., Böttger E.C. \& Decker T. 1993. The gene encoding IFP 53/tryptophanyl-tRNA synthetase is regulated by the gamma-interferon activation factor. J. Biol. Chem. 268:590-595.
Sugimoto H., Oda S., Otsuki T., Hino T., Yoshida T. \& Shiro Y. 2006. Crystal structure of human indoleamine 2,3-dioxygenase: catalytic mechanism of 02 incorporation by a heme-containing dioxygenase. Proc. Natl Acad. Sci. USA 103:611-616.

Suzuki S., Toné S., Takikawa O., Kubo T., Kohno I. \& Minatogawa Y. 2001. Expression of indoleamine 2,3-dioxygenase and tryptophan 2,3-dioxygenase in early concepti. Biochem. J. 355:425-429.

Szekeres-BarthoJ.\&Wegmann T.G. 1996. A progesterone-dependentimmunomodulatory protein alters the Th1/Th2 balance. J. Reprod. Immunol. 31:81-95.

Tanriverdi F., Silveira L.F., MacColl G.S. \& Bouloux P.M. 2003. The hypothalamic-pituitary-gonadal axis: immune function and autoimmunity. J. Endocrinol. 176:293-304.

Xiao B.G., Liu X. \& Link H. 2004. Antigen-specific T cell functions are suppressed over the estrogen-dendritic cell-indoleamine 2,3-dioxygenase axis. Steroids 69:653-659. 